\title{
What is really being lost in wormholes?
}

\author{
P. F. González-Díaz \\ Instituto de Optica "Daza de Valdés," Consejo Superior de Investigaciones Científicas, \\ Serrano 121, 28006 Madrid, Spain \\ (Received 15 January 1990; revised manuscript received 18 June 1990)
}

\begin{abstract}
We discuss a new general method to obtain the Tolman-Hawking wormhole instanton by simply providing the Robertson-Walker manifold with an extra three-sphere with a minimum radius equal to the Planck length. This topology change does not imply any unphysical value of the effective gravitational constant and requires a total energy which is no longer zero. It is argued that, even for wormholes with zero total energy, there is no loss of quantum coherence in the observable matter sector of flat spaces. The possibility that wormholes can induce chaotic behavior of spacetime is discussed by using a quantum-optical interpretation for wormhole interactions. Also discussed in the light of this interpretation is the Coleman mechanism for the vanishing of the cosmological constant and the fixing of physical coupling constants. A value zero is kept as the most probable value of the cosmological constant for large universes, but it is argued that the Coleman mechanism should be reinterpreted for small universes or the fixing of the coupling constants. It is also shown that the Wheeler-DeWitt equation for wormholes or little baby universes is reducible to a Klein-Gordon wave equation and that some of the solutions should be associated with antiwormholes, i.e., nontrivial topological changes with negative energy.
\end{abstract}

\section{INTRODUCTION}

Spatial topological changes in quantum gravity have been described by a path integral over Euclidean fourgeometries. ${ }^{1}$ The recent discovery of some gravitational instantons that implement these topology changes or wormholes has uncovered a number of unexpected features which may lead to drastic changes concerning general and deep views in physics. ${ }^{2}$ A wormhole is a microscopic contact between two otherwise smooth arbitrarily distant regions of spacetime. What is particularly interesting about these topological changes is that they may provide us for the first time with a suitable link between the smallest and largest distance scales. ${ }^{3}$

In the last few years there has been some controversy concerning whether quantum coherence is lost when Planck-sized baby universes resulting from wormholes branch off from one large asymptotically flat parent universe. $^{4-10}$ The idea was originally suggested by Hawking who realized ${ }^{11}$ that in non-Minkowskian spacetimes any correlation between two given systems is irretrievably lost whenever one of them gets beyond the horizon of the other. The implications of this idea also prompted some vigorous opposition. ${ }^{12}$ Recently, Hawking has given the idea a new form. In several papers, ${ }^{4,5,13}$ which supersede earlier work, he associates loss of quantum coherence with wormholes rather than with simply connected spacetimes. His theory of wormholes has been complemented by work done by Giddings and Strominger $^{6}$ who found wormholes as actual instantons in a theory of gravity coupled to massless axions. Also supporting the Hawking proposal is some work by Lavrelashvili, Rubakov, and Tinyakov. ${ }^{7,14}$

However, further consideration on the effective interaction of wormholes with observable matter in asymp- totic flat spaces ${ }^{4,5}$ allowed Coleman ${ }^{8}$ and Giddings and Strominger ${ }^{9}$ to argue that the production of baby universes does not lead to any loss of quantum coherence in the matter-field sector. Coleman showed ${ }^{8}$ that by performing a large series of measurements the initial family wave function collapses into a certain state (a $q$ state) which remains invariant during the interaction, so that there is no loss of quantum coherence induced by changes in topology. Hawking and Laflamme have argued ${ }^{13}$ that it is rather metaphysical to discuss whether the presence of little closed universes leads to a loss of quantum coherence or merely a lack of knowledge of the initial state.

Coleman has nevertheless considered the effects of wormholes in the Euclidean path-integral formalism of quantum gravity from a different point of view. Using an earlier idea due to Hawking, ${ }^{15}$ he has suggested the remarkable idea that the entire effect of wormholes is to modify the coupling constants in such a way that the probability for a given value of the cosmological constant is infinitely peaked at zero. It appears that the branching off of little baby universes from flat spaces leads to the introduction of vacuum parameters for low-energy coupling constants which, in this way, are hoped to be determined dynamically.

However, all of these claims must confront an initial basic problem. Wormhole solutions occur only for certain special types of matter fields, such as axionic or conformally coupled fields; how then could these restricted instantons affect issues so universal as the vanishing of the cosmological constant or the loss of quantum coherence? On the other hand, Halliwell and Laflamme have recently shown ${ }^{16}$ that the Euclidean solution for a scalar field conformally coupled to gravity, representing a Tolman-Hawking wormhole, ${ }^{17}$ can give rise to antigravitational regimes where the effective gravitational con- 
stant becomes negative. The problem is aggravated even more because Ruback has proven ${ }^{18}$ a uniqueness theorem for wormhole spaces with vanishing Ricci scalar according to which, among all candidate metrics and manifolds, the unique extremum of the Euclidean action is the Tolman-Hawking wormhole.

Recently, general wormhole solutions of the TolmanHawking kind, which do not induce any unphysical value of the effective gravitational constant, have been obtained ${ }^{19}$ from pure gravity by simply introducing a cutoff in the scale factor squared; this procedure is equivalent to provide the Robertson-Walker manifold with an extra three-sphere with minimum radius of the order of the Planck scale. The main aim of this paper is to reexplore the effects produced in low-energy particle physics by the interaction with wormholes using this general wormhole instanton.

The outline of the paper is as follows. In Sec. II we review wormhole spacetimes having the analytical structures discussed by Giddings and Strominger, and Halliwell and Laflamme. Section III contains a detailed discussion in terms of second-order correlation functions of the loss of quantum coherence induced by wormholes. It is concluded that, even for massless baby universes, wormholes would not induce any loss of quantum coherence, but a new general restriction in our capability of getting information from any physical system. In Sec. IV we point out the modifications to be expected in the wave equation for wormholes if we introduce a cutoff in the scale factor squared and advance a specific wormholeinduced quantum-optical scenario where the spacetime can show chaotic behavior. Section $\mathbf{V}$ discusses the Coleman mechanism for the vanishing of the cosmological constant and the fixing of physical coupling constants. It is argued that the Coleman procedure is essentially valid for the part of the cosmological constant originating its macroscopic role, but it is not for those parts of the cosmological constant associated with its role as a microscopic coupling constant, which are expected to have dominated at the earliest stages in the evolution of the Universe, or for the fixing of the coupling constants. The results are summarized, adding some comments, in Sec. VI. We add three Appendixes. In Appendix A we briefly review the Lorentzian solutions corresponding to the Tolman-Hawking wormhole studied in this paper. Several arguments in favor of the used correspondence principle for the scalar field momentum are given in Appendix B. Appendix $\mathrm{C}$ deals with the derivation and interpretation of a Klein-Gordon equation for wormholes. A Dirac equation for wormholes is also briefly discussed.

\section{WORMHOLE SPACETIMES}

We review here wormhole spacetimes obtained as solutions to the Euclidean equations of motion corresponding to coupling either a three-index axionic field ${ }^{6}$ (which is equivalent to a purely imaginary minimally coupled scalar field) or a conformal scalar field ${ }^{16}$ to Einstein gravity, as well as a semiclassical solution obtained from pure gravity. ${ }^{19}$ Yang-Mills solutions ${ }^{20}$ are not local minima of the action, and it is therefore not clear that they contribute to the semiclassical approximation and will not be considered in this work. There are also Kantowski-Sachs wormholes $^{21}$ and general axionic wormholes which were derived $^{22}$ from a gravity theory containing higherderivative terms $R^{2}$. However, we will restrict ourselves to the simplest case of Hilbert-Einstein gravity for a four-dimensional isotropic minisuperspace Euclidean metric of the form

$$
\begin{aligned}
d s^{2} & =N^{2} d \tau^{2}+a^{2}(\tau) d \Omega_{3}^{2} \\
& =a^{2}(\eta)\left(N^{2} d \eta^{2}+d \Omega_{3}^{2}\right),
\end{aligned}
$$

where $N$ is the lapse function and $d \Omega_{3}^{2}$ is the metric on the three-sphere.

A generic Euclidean action integral describing both Hilbert-Einstein gravity coupled to an axionic field and a conformally coupled scalar field can be written in the form $^{16,23}$

$$
\begin{aligned}
\widetilde{I}= & -\frac{1}{16 \pi G} \int_{M} d^{4} x g^{1 / 2}\left[\left(1-8 \pi G \xi \Phi^{2}\right) R-2 \lambda\right] \\
& +\frac{1}{2}(-1)^{2 n} \int_{M} d^{4} x g^{1 / 2}(\nabla \Phi)^{2} \\
& -\frac{1}{8 \pi G} \int_{\partial M} d^{3} x h^{1 / 2}\left(1-8 \pi G \xi \Phi^{2}\right) K,
\end{aligned}
$$

where $\lambda$ denotes a cosmological constant, and $h$ and $K$ are, respectively, the determinant of the first fundamental form and the trace of the second fundamental form,

$$
K_{a b}=\frac{\partial h_{a b}}{2 N \partial \tau},
$$

of the boundary $\partial M$ of the manifold $M$.

The Tolman-Hawking wormhole corresponds to the conformally coupled field case $\xi=\frac{1}{6}, n=1$, and the Giddings-Strominger wormhole corresponds to the minimally coupled axionic imaginary field case $\xi=0$, $n=\frac{1}{2}$.

Variation with respect to $a, \Phi$, and $N$ of the minisuperspace Euclidean action obtained by inserting (2.1) into (2.2) yields the field equations for the scale factor and scalar field, and the constraint equation. For $\xi=0, n=\frac{1}{2}$, it is obtained as

$$
\begin{aligned}
& a^{3} \dot{\Phi}=K_{0} N, \\
& \frac{1}{2} a^{\prime 2}+U\left(a, \hat{K}_{0}\right)=0, \\
& a^{\prime \prime}=-\partial U\left(a, \hat{K}_{0}\right) / \partial a,
\end{aligned}
$$

where

$$
\hat{K}_{0}=(4 \pi G / 3)^{1 / 2} K_{0},
$$

$K_{0}$ is an integration constant, and

$$
U\left(a, \hat{K}_{0}\right)=\frac{1}{2}\left[\frac{\hat{K}_{0}^{2}}{a^{2}}+\frac{\lambda a^{4}}{3}-a^{2}\right] .
$$

The four-dimensional Giddings-Strominger wormhole is then given by

$$
a=\widehat{K}_{0} \cosh ^{1 / 2}(2 \eta),
$$

corresponding to the case $\lambda=0$. 
For $\xi=\frac{1}{6}, n=1$, we obtain

$$
\chi^{\prime 2}-\chi^{2}=M^{2},
$$

where $\chi=(4 \pi G / 3)^{1 / 2} a \Phi$ and $M^{2}$ is an integration constant. The constraint and equation of motion for the scale factor $a$ are given by Eqs. (2.4b) and (2.4c), with $U\left(a, \hat{K}_{0}\right)$ being replaced by $U(a, M)$ :

$$
U(a, M)=\frac{1}{2}\left(M^{2}+\lambda a^{4}-a^{2}\right) .
$$

Equations $(2.4 \mathrm{~b})$ and $(2.4 \mathrm{c})$ represent the motion of a particle in a potential $U$ with zero total energy. The Tolman-Hawking wormhole corresponds then to a particular solution of the equations of motion and constraint associated with the conformally coupled scalar field for $\lambda=0$ :

$$
\chi=a=M \cosh \eta \text {. }
$$

The problem with the gravitational constant for this kind of wormhole is now easily seen. From (2.2) one obtains for $\xi=\frac{1}{6}, n=1$,

$$
G_{\mathrm{eff}}=G\left[1-(\chi / a)^{2}\right]^{-1}
$$

inserting (2.9) into (2.10), it follows that $G_{\text {eff }}$ is infinite along the complete interval of $\eta$ values. For the most general wormhole solution obtained by Halliwell and Laflamme, it can be checked that the value of the effective gravitational constant has to become necessarily negative for some relevant interval of values of the conformal time $\eta$.

Four-dimensional spacetimes having exactly the same analytical structure as the Tolman-Hawking wormhole have been recently obtained in the absence of any matter field by introducing a three-sphere with minimal constant radius of the order the Planck length, $m$, in the usual Robertson-Walker isotropic manifold (2.1), ${ }^{19}$ according to

$$
a^{2} \rightarrow a^{2}-m^{2}
$$

The inclusion of such a cutoff in the scale factor squared is motivated by recent work done in quantum gravity ${ }^{24,25}$ which predicts that it is altogether impossible to get a spacetime resolution better than the Planck length. In fact, by quantizing the conformal factor $\Omega$ in a manifold provided with the conformal metric

$$
g_{a b} \rightarrow \Omega^{2} g_{a b},
$$

it has been found ${ }^{25}$ that the introduction of a maximum resolution limit in $a, \Delta a \geq m$, amounts to a modification of the scale factor squared given precisely by $a^{2} \rightarrow a^{2}-m^{2}$. Even when looked at as semiclassical solutions, wormholes must contain a certain level quantization above that which is implied by Euclidean gravity. A prescription for the extent to which such a quantization should be done is not at present generally known. For example, axionic wormholes are obtained by demanding a fixed axionic charge. Here wormhole solutions should be interpreted as flat-spacetime solutions, to which one adds the physical consequences of quantizing the metric conformal factor $\Omega$. In the end, since the most probable value of the wormhole neck size is about the minimal uncertainty in the scale factor $a$, one could interpret a wormhole as a topological consequence from the existence of a finite maximum quantum resolution limit for the determination of the scale factor $a$. This is equivalent to introducing a transformed Euclidean time of the form

$$
d \tau^{\prime}=\left(1-\frac{m^{2}}{a^{2}}\right)^{1 / 2} d \tau .
$$

Performing the above transformation, the Euclidean action for a scalar field conformally coupled to gravity becomes

$$
\begin{aligned}
\widetilde{I}=\frac{1}{2} \int d \tau^{\prime} a N & -\frac{\dot{a}^{2}}{N^{2}}-1+\lambda a^{2}-\lambda m^{2} \\
& \left.+\frac{\dot{\chi}^{2}}{a^{2} N^{2}}\left(a^{2}-m^{2}\right)+\frac{\chi^{2}}{a^{2}-m^{2}}\right),
\end{aligned}
$$

where now $\dot{a}=d a / d \tau^{\prime}, \dot{\chi}=d \chi / d \tau^{\prime}$, and the Euclidean region is defined for $a \geq m$. Variation with respect to $N$, $\chi$, and $a$ now produces

$$
\begin{aligned}
& a^{\prime 2}-\left(1+2 m^{2} \lambda\right) a^{2}+m^{2}\left(1+\lambda m^{2}\right)+\lambda a^{4} \\
& -\chi^{\prime 2}\left(1-\frac{m^{2}}{a^{2}}\right)+\chi^{2}=0, \\
& \chi^{2}-\chi^{\prime 2}\left(1-\frac{m^{2}}{a^{2}}\right)=M^{2}, \\
& \left(1-3 \lambda\left(a^{2}-m^{2}\right)-\frac{\chi^{\prime 2}}{a^{2}}-2 \frac{\alpha^{\prime \prime}}{a}\right)\left(a^{2}-m^{2}\right) \\
& +a^{\prime 2}+\chi^{2}=0,
\end{aligned}
$$

where we have adopted the gauge $N=1$, and $a^{\prime}=d a / d \eta$.

Again, we are dealing with a physical situation which is representable as the motion of a particle in a given potential; in this case, however, the total energy of the system is no longer zero. This is most clearly seen by considering the simplest wormhole case where $\lambda=0$, for which Eq. (2.13a) reduces to

$$
-\frac{1}{2} a^{\prime 2}-U(a, M)=m^{2},
$$

where $U(a, M)$ is given by $(2.8)$ for $\lambda=0$.

Furthermore, this remarkable consequence constitutes by itself a physical justification for our procedure. An observer in the asymptotic region does not know anything about the values of the three-metric $h_{i j}$ and the matter field $\phi$ on any cross section $S$ of the wormhole. Therefore, as a necessary additional quantization step which would be introduced prior to the usual pathintegral Euclidean quantization, one should sum over all possible values of $h_{i j}$ and $\phi$ on that cross section. This would ultimately mean that the two four-geometries are joined at $S$, so that the three-surface would no longer divide the whole manifold into two disconnected parts. Therefore, one should represent the quantum state of the wormhole by a density matrix rather than a pure-state wave function. The point now is that the WheelerDeWitt equation for a density matrix is no longer equal 
to zero, but to a nonzero residual energy, which is precisely $m^{-1}$ for the pure gravity case. It is in this sense that the introduction of a UV cutoff in the scale factor squared should be interpreted as reflecting the physical feature that an observer in the asymptotic region cannot know anything about the values taken by the relevant degrees of freedom on any cross section of the wormhole.

Thus, representing the quantum state of wormholes by a density matrix and introducing a cutoff in the scale factor, and then representing the wormhole state by a wave function, could be considered as two, to some extent equivalent alternate ways for dealing with quantum wormholes properly. In this paper we shall concentrate mostly on the last representation, leaving a full account of the first one for a forthcoming publication. ${ }^{26}$

The solution to $(2.14)$ is still given by the TolmanHawking wormhole

$$
a=\left(M^{2}+m^{2}\right)^{1 / 2} \cosh \eta,
$$

or, in Robertson-Walker time $\tau$,

$$
a=\left(M^{2}+m^{2}+\tau^{2}\right)^{1 / 2} .
$$

In this case the wormhole throat radius is given by $\left(M^{2}+m^{2}\right)^{1 / 2}$.

For $\lambda>0$, we obtain the Euclidean solution in terms of the Robertson-Walker time $\tau$ :

$$
\begin{gathered}
a(\tau)=(2 \lambda)^{-1 / 2}\left[1+2 m^{2} \lambda-\left(1-4 \lambda M^{2}\right)^{1 / 2}\right. \\
\left.\times \cos \left(2 \lambda^{1 / 2} \tau\right)\right]^{1 / 2}
\end{gathered}
$$

which lies in the region $a_{-}<a<a_{+}$defined by

$a_{ \pm}=(2 \lambda)^{-1 / 2}\left[1+2 m^{2} \lambda \pm\left(1-4 \lambda M^{2}\right)^{1 / 2}\right]^{1 / 2}$.

A discussion of the Lorentzian solutions corresponding to (2.15) and (2.16) can be found in Appendix A.

Solutions (2.15) and (2.16) have an analytical structure similar to that of those obtained by Halliwell and Laflamme, ${ }^{16}$ and reduce identically to them in the classical limit $m \rightarrow 0$. There is, however, a crucial difference. Solutions (2.15) and (2.16) no longer lead to any problem with the gravitational constant. This can be readily seen by considering again the case $\lambda=0$ for which we obtain, for the equation of motion of the field $\chi$,

$\left(m^{2}+M^{2}\right)\left(\chi^{2}-\chi^{\prime 2}-M^{2}\right) \cosh ^{2} \eta+m^{2} \chi^{\prime 2}=0$.

A solution to $(2.18)$ is

$$
\chi=i\left(m^{2}+M^{2}\right)^{1 / 2} \sinh \eta \text {. }
$$

From (2.10) and (2.19) we have

$$
G_{\mathrm{eff}}=G\left(1+\tanh ^{2} \eta\right)^{-1}>0 .
$$

Actually, the value of the effective gravitational constant varies from $\frac{1}{2} G$ for $\eta \rightarrow \pm \infty$ to $G$ at the wormhole throat $(\eta=0)$ for the particular Tolman-Hawking wormhole (2.15). A similar result can be obtained for a nonzero cosmological constant. In order to interpret this result, one would resort to the above-mentioned previous quantization step for wormholes. It could well be that the infinite value taken by the effective gravitational con- stant in current conformally invariant scalar field wormholes is nothing but an artifact arising from not introducing such a previous quantization step (i.e., not recognizing any uncertainty about the baby universe state). Once that uncertainty is explicitly introduced in the action of the system, the problem with the effective gravitational constant is no longer present in the theory. One more technical reason may be found by generalizing to the density-matrix representation of the quantum state of wormholes. The contribution from wormholes to the action in the path integral is generally given by a bilocal effective Lagrangian, ${ }^{4,5}$ which, in the case of a density matrix, would be weighted by a probability factor given by the inverse of the total wormhole energy. If, as happens in the current interpretation, ${ }^{16}$ the total wormhole energy is zero, convergence in the wormhole contribution could be achieved only at the cost of having an infinite effective gravitational constant. Since in our procedure the total wormhole energy is different from zero (i.e., the wormhole is not on shell), in order for the wormhole contribution to be finite, we no longer require $G_{\text {eff }}$ to be infinite.

It is worth noticing that we have ended up with a pure imaginary scalar field which makes wormholes obtained from a conformally coupled scalar field to some extent equivalent to those obtained from an axionic field. The scalar field $\Phi$ associated with $\chi$ would correspond to the solution of an equation of motion of the form

$$
a^{2} \Phi^{\prime}=\text { const }=i\left(m^{2}+M^{2}\right)^{1 / 2}\left(\frac{3}{4 \pi G}\right)^{1 / 2},
$$

which has the same form as that for a massless minimally coupled axion expressed as a purely imaginary scalar field. It is in this sense that the obtained wormhole behaves as though it were a hybrid of Tolman-Hawking and Giddings-Strominger wormholes, whereas if its spacetime analytical structure were that of Tolman and Hawking, the involved matter field would be axionic. This kind of equivalence does not mean that the wormhole obtained in this work is the same as that obtained by Giddings and Strominger. It seems clear that in the latter case the wormhole is stabilized by a conserved global charge of the axionic field, whereas there is no such a conserved charge in the solution obtained here.

On the other hand, the limit $M \rightarrow 0$ (no matter field) leaves us with a wormhole ${ }^{19} a=m \cos \eta$. This indicates that the Tolman-Hawking wormhole can be considered as a general solution for pure gravity. ${ }^{19}$ In this case the value of the effective gravitational constant does coincide with the Newtonian value for the entire conformal time interval. It is in this sense that one could interpret the existence of well-defined wormholes as an immediate consequence from the impossibility of performing measurements of the spacetime position with a precision greater than about the Planck length. Furthermore, since, according to (2.14), the total energy corresponding to a baby universe is not zero in the picture we are considering, creation (annihilation) of a wormhole at a given point in the observable asymptotically flat region will then destroy (generate) a given amount of matter confor- 
mally coupled to gravity at that point. Thus any large universe endowed with wormholes connecting it to other large universes will become a dissipative system.

Before ending this section we want to note the remarkable relation between the scale factor and the conformally coupled scalar field arising from (2.15) and (2.16):

$$
a^{2}+\chi^{2}=m^{2}+M^{2} .
$$

Such a relation is preserved in the Lorentzian region and might be viewed as the fundamental equation defining the Tolman-Hawking wormhole considered in this work. In the case $M=0$, Eq. (2.21) holds still for the quantum constant $\mathrm{m}^{2}$. This would ultimately imply that the introduction of a finite-resolution limit is somewhat equivalent to assuming the presence of a residual scalar quantum field (for more about this, see Appendix C).

\section{WHEELER-DeWITT EQUATION AND THE LOSS OF QUANTUM COHERENCE}

As pointed out by Hawking, ${ }^{5}$ if we use the dilute wormhole approximation, the effect of wormholes on background matter fields $\phi$ in asymptotically flat spaces is given by the wormhole-filtered Green's function

$\left\langle 0\left|\phi\left(x_{1}\right) \cdots \phi\left(x_{n}\right)\right| \Psi\right\rangle\left\langle\Psi\left|\phi\left(y_{1}\right) \cdots \phi\left(y_{m}\right)\right| 0\right\rangle$,

where $\phi$ represents a conformally invariant scalar field, $x_{1}, \ldots, x_{n}$ and $y_{1}, \ldots, y_{m}$ are points in the two asymptotically flat regions connected by wormholes, and $|\Psi\rangle$ denotes the quantum state of a baby universe or wormhole on a three-surface $S$, which is a cross section of the wormhole. The function (3.1) can be computed by path integrating over all matter fields $\phi$ and all wormhole metrics. The quantum state $|\Psi\rangle$ is described by a wave function $\psi$, which depends on the induced metric $h_{i j}$ and the values $\phi_{0}$ of the matter fields on the three-surface $S$ and should satisfy the corresponding Wheeler-DeWitt equation. In the minisuperspace model we have used, assuming that there is no indeterminacy in the scale factor, the Wheeler-DeWitt equation is obtained by applying the correspondence principle to the constraint

$$
-a^{\prime 2}+a^{2}+\chi^{\prime 2}-\chi^{2}=0 \text {. }
$$

The prescription $a^{\prime} \rightarrow \partial / \partial a, \chi^{\prime} \rightarrow \partial / \partial \chi$, leads to a Wheeler-DeWitt equation (modulo factor ordering):

$$
\left(-\frac{\partial^{2}}{\partial a^{2}}+\frac{\partial^{2}}{\partial \chi^{2}}+a^{2}-\chi^{2}\right) \psi=0 \text {. }
$$

Here $\psi$ should be interpreted as the quantum state of the wormhole.

Starting with (3.1) and (3.3), it was first shown by Hawking $^{5}$ and confirmed later by Coleman ${ }^{10}$ that the branching off of little closed universes would affect lowenergy particle physics in the asymptotically flat region of the universe which we live in just as if there were effective interactions in flat space of the general form

$$
L^{\mathrm{eff}} \propto B_{i}(\phi)\left(b_{i}^{\dagger}+b_{i}\right),
$$

where $B_{i}(\phi)$ is a polynomial in the field operators $\phi$, and $b_{i}^{\dagger}, b_{i}$ are independent second quantization Fock operators for the little closed universe sector.

There is a point in the Hawking procedure for deriving Eq. (3.4) which we would like emphasizing. It is that, although the Wheeler-DeWitt equation (3.3) obviously has harmonic-oscillator solutions in the two variables $a$ and $\chi$, there is a problem with the zero-point energies of each scale factor harmonic-oscillator mode. Such as it was formulated, Hawking theory did not provide for any proper mechanism to cancel these zero-point energies, and one has to assume them to be eventually canceled or subtracted by fermions in a supersymmetric theory.

Although this formalism is for wormholes obtained from a scalar field conformally coupled to gravity, one can always use the freedom to establish an equivalence between the conformal and minimal scalar fields coupled to gravity, ${ }^{27}$ so that it is always possible to adapt the present formalism to the case of an axionic wormhole.

Let us now consider the Hawking proposal ${ }^{4,5,13}$ that wormholes induce loss of quantum coherence in the matter-field sector of the theory. Loss of quantum coherence may come about when we introduce independent Fock-space bases for matter fields $\left|\alpha_{i}\right\rangle$ and wormholes $\left|\beta_{j}\right\rangle$, so that if no closed universes were initially present, the initial state for the full system would be written as $\left|\alpha_{i}\right\rangle|0\rangle$. Basically Hawking's argument now runs as follows. Since an observer in the flat region cannot measure the states of the closed universes, one should sum over all possible states for such universes; this would produce a mixed final state. However, although in principle this would lead to loss of information about the state of the observable subsystem, concerning the fate of quantum coherence, one should elaborate a little bit further the argument before reaching any definitive verdict about it.

There would be a loss of quantum coherence if, and only if, after the interaction, the matter field $\phi$ took on less correlated values at the two distantly separated points where the wormhole joins flat space. In order to discuss this in more quantitative terms, we introduce $n$ th-order normalized correlation functions ${ }^{28}$ for the matter field,

$$
g^{(n)}\left(x_{1}, \ldots, x_{2 n}\right)=\frac{G^{(n)}\left(x_{1}, \ldots, x_{2 n}\right)}{\prod_{j=1}^{2 n}\left[G^{(1)}\left(x_{j}, x_{j}\right)\right]^{1 / 2}},
$$

in which, if we expand $\phi$ in terms of Fock operators $c$ and $c^{\dagger}$, we have, for the unnormalized function $G^{(n)}$,

$$
\begin{aligned}
G^{(n)}\left(x_{1}, \ldots, x_{2 n}\right)=\operatorname{Tr}[ & \rho_{\phi} c^{\dagger}\left(x_{1}\right) \cdots c^{\dagger}\left(x_{n}\right) \\
& \left.\times c\left(x_{n+1}\right) \cdots c\left(x_{2 n}\right)\right],
\end{aligned}
$$

with $\rho_{\phi}$ being the density matrix for the $\phi$ sector of the quantum state.

The field $\phi$ will be coherent $^{28}$ when $\left|g^{n}\left(x_{1}, \ldots, x_{2 n}\right)\right|=1$, for $n=1,2, \ldots$, and this requires factorization of the correlation function. This is achieved by introducing the so-called coherent states $|\zeta\rangle$, which have minimal quantum uncertainty and satisfy

$$
b|\zeta\rangle=\xi|\xi\rangle,\langle\xi| b^{\dagger}=\langle\xi| \zeta^{*} .
$$

The first-order quantum coherence $g^{(1)}$ does not essen- 
tially differ from the corresponding classical quantity and is always unity for single-mode states. It is the secondorder quantum coherence $g^{(2)}$ which may separate from its classical counterpart and take on values other than unity for single-mode coherent states. Change (loss or gain) of quantum coherence is associated with processes in which the second-order quantum coherence function $g^{(2)}$, which is unity for single-mode coherent states, undergoes a given variation. A typical way by which this function may vary for a single mode is when an initially pure state transforms into a mixed state. We shall show that this kind of evolution cannot take place for the matter field for an interaction Hamiltonian derived from (3.4).

Consider the simple example in which $B_{i}(\phi) \propto m_{P}^{2} \phi^{2}$, where $m_{P}$ denotes the Planck mass. The interaction Hamiltonian will have the form

$$
H_{\mathrm{int}} \propto m_{P} \sum_{i} c^{\dagger} c\left(a_{i}^{\dagger}+a_{i}\right),
$$

where we have assumed that the mass of the scalar field $\phi$ gets a value near $m_{P}$ while interacting with the wormhole. $^{5}$

Starting with the equation of motion for the density matrix $\rho$ of the field system, which we then iterate ${ }^{29}$ for small increment of time, and assuming

$$
\rho(t)=\rho_{\phi}(t) \prod_{i} \rho_{\omega i}(0),
$$

after tracing over the closed universe sector, we can get, with the same approximation as used in ordinary timedependent perturbation calculations,

$$
\begin{gathered}
\dot{\rho}_{\phi}(t)=-\delta m_{P}\left(c^{\dagger} c c^{\dagger} c \rho_{\phi}-2 c^{\dagger} c \rho_{\phi} c^{\dagger} c\right. \\
\left.+\rho_{\phi} c^{\dagger} c c^{\dagger} c\right),
\end{gathered}
$$

where $\delta$ is merely an undetermined numerical coefficient whose value will depend on the number of closed universes initially present.

Taking matrix elements in the Fock space of scalar particle number states, $P_{n, m}=\left\langle n\left|\rho_{\phi}(t)\right| m\right\rangle$, we solve (3.9):

$$
P_{n, m}(t)=P_{n, m}(0) \exp \left[-\delta m_{P} t(n-m)^{2}\right] .
$$

Changes of the statistical properties of the matter field are only then possible for off-diagonal matrix elements. The correlation functions and moments are obtained, however, from the expectation values of the scalar particle numbers which are evaluated tracing over these numbers. In particular, the quantum degree of second-order coherence, expressed in terms of the mean and meansquared single-mode scalar particle number,

$$
g^{(n)}=\frac{\overline{\alpha^{2}}-\bar{\alpha}}{\overline{\alpha^{2}}}
$$

will not change during interaction (3.8). For an initially coherent quantum field $P_{n, m}^{\text {coh }}(0),(3.10)$ can be written in the form

$$
\begin{aligned}
P_{n, m}(t)= & \exp \left[-|\zeta|^{2}-\delta m_{P} t\left(n^{2}+m^{2}\right)\right] \\
& \times\left[\zeta \exp \left(\delta m_{P} m t\right)\right]^{n} \\
& \times\left[\zeta^{*} \exp \left(\delta m_{P} n t\right)\right]^{m}(n ! m !)^{-1 / 2},
\end{aligned}
$$

which reduces to

$$
P_{n}^{\mathrm{coh}}(0) \equiv \exp \left(-|\xi|^{2}\right)|\xi|^{2 n} / n !,
$$

for $n=m$. Thus, even if the system were initially in a coherent state, there would be no observable variation of the particle state, loss of quantum coherence, or violation of any classical statistical properties. The argument can be straightforwardly generalized for any initial state of the matter system.

This result is related to the Coleman interpretation of the loss of available information as a red herring. ${ }^{8}$ Coleman argues that the demand of locality on parent universes implies that the operators $Q_{i}=a_{i}^{\dagger}+a_{i}$ must all commute, and hence one can introduce $q_{i}$ states $|q\rangle$, so that

$$
Q_{i}\left|q_{i}\right\rangle=q_{i}\left|q_{i}\right\rangle,
$$

which means that, if the closed universe sector of the quantum state were initially in a $q_{i}$ state, the effective interaction (3.4) would leave it in the same state. It follows that the net effect of the little closed universes is to introduce some undetermined parameters $q_{i}$. The point now is to realize that, since closed universes cannot be detected by an observer in the flat region of the space, the initial quantum state cannot be a single $q$ state, but there must be a total spread of values of $q$. The physical reason for this is that the second-order quantum coherence in Fock space for an initial single-mode pure state,

$$
g_{i}^{(2)}=\left(\alpha_{i}-1\right) \alpha_{i}^{-1},
$$

would necessarily be different from that corresponding to the final single-mode mixed state, which, for a density matrix

$$
\rho_{\phi f}=\beta_{1}\left|\alpha_{i}\right\rangle\left\langle\alpha_{i}\left|+\beta_{2}\right| \alpha_{f}\right\rangle\left\langle\alpha_{f}\right|,
$$

is given by

$$
g_{f}^{(2)}=\frac{\beta_{1} \alpha_{i}\left(\alpha_{i}-1\right)+\beta_{2} \alpha_{f}\left(\alpha_{f}-1\right)}{\beta_{1} \alpha_{i}^{2}+\beta_{2} \alpha_{f}^{2}},
$$

with $\beta_{1}+\beta_{2}=1$ and $\left|\alpha_{i}\right\rangle \neq\left|\alpha_{f}\right\rangle$. However, we have shown above that the interaction with baby universes leaves $g^{(2)}$ unchanged, so that such an interaction can by no way induce any transition between pure and mixed states or change of quantum coherence for the considered single-mode scalar field. This argument ends our proof that wormholes do not induce any loss of quantum coherence. The proof can be extended to the case of more complicated matter field or wormhole configurations. Although, of course, it is relatable to the Coleman line of reasoning, our procedure is technically and methodologically different of the Coleman method, and uses the more transparent quantum-optical concepts required to investigate any subject related to the maintainence of quantum coherence. 
As to the interpretation of this result, it follows from the discussion above that the only way to recover invariance in $g^{(2)}$ is by assuming $\rho_{\phi i}=\rho_{\phi f} \Longrightarrow$ mixed state. All our arguments amount to a lack of knowledge about the initial state. Doing an infinite number of identical experiments for determining with full accuracy the parameters $q_{i}$ is therefore equivalent to tracing the final state over the different values of $q$ : There is no real loss of information here with respect to the initial state because the information that supposedly is being lost was already missing initially, or alternatively, the lack of part of the information about the state of any observable system due to interaction with wormholes can be also attributed to the feature that, even when there is no matter in the asymptotic region, wormholes do exist and are themselves in mixed states describable by a density matrix. Through the effective interaction between such wormholes and the observable matter, the uncertainty about the state of wormholes is somehow instantly transferred to all kind of observable matter in the asymptotic region. Also, the vacuum free energy corresponding to all matter fields should reflect-or, rather, may be a direct consequence from-the uncertainty in the pure gravity wormholes whose existence is assumed here.

Quantum gravity seems thus to preclude the observation of any really pure state at any scale of energy, and this would mean an extra degree of uncertainty in all imaginable physical situations. What we are assuming in this paper is that this extra degree of uncertainty (above that which is implied by Heisenberg indetermination principle) could be interpreted as a finite, essentially quantum maximum-resolution limit for the determination of the spacetime location in any conceivable experiment.

\section{QUANTUM WORMHOLES: A ROUTE TO CHAOTIC SPACETIME?}

The analysis in the preceding section is based on the Wheeler-DeWitt equation (3.3) for a conformally coupled scalar field, assuming that there is no indeterminacy in the scale factor. As discussed in Sec. II, this picture is ill defined in at least two respects: (1) The kind of wormholes used induce infinite or even negative values for the gravitational constant, and (2) the theory is defined only for special kinds of matter fields and has therefore a restricted applicability. There are other problems with Eq. (3.3) that are general shortcomings of the Wheeler-DeWitt picture and have been discussed elsewhere. ${ }^{30}$ It has been also stated that the introduction of a nonzero quantum indeterminacy in the scale factor may cure the two above-mentioned problems. In this section we shall consider the effects of wormholes on matter fields $\phi$ in the asymptotically flat region by assuming a wormhole spacetime as that is given by Eq. (2.15). In this case the constraint equation is [cf. Eq. (2.14)]

$$
-a^{\prime 2}+a^{2}+\chi^{\prime 2}\left(1-\frac{m^{2}}{a^{2}}\right)-\chi^{2}=m^{2},
$$

whose right-hand side (RHS) is to be interpreted as a purely gravitational quantum term of the order the Planck scale.
We first obtain the wave equation that corresponds to (4.1) by noting that, since we have not explicitly introduced any cutoff for the length $\chi$, if we choose the momentum operator for $a^{\prime}$ to be $\partial / \partial a$, the classical quantity to be associated with the momentum operator $\partial / \partial \chi$ has to be $d \chi / d \eta^{\prime}$ rather than $\chi^{\prime}=d \chi / d \eta$, with $\eta^{\prime}$ being the transformed Euclidean conformal time. More compelling reasons for this choice are given in Appendix B. Now, just as Eq. (2.11) may be derived by defining transformation-invariant conformal time

$$
d \eta=\frac{d \tau}{a}=\frac{d \tau^{\prime}}{\left(a^{2}-m^{2}\right)^{1 / 2}},
$$

we can obtain a relation linking $\eta$ to $\eta^{\prime}$ by defining transformation-invariant Robertson-Walker time

$$
d \tau=a d \eta=\left(a^{2}-m^{2}\right)^{1 / 2} d \eta^{\prime}
$$

i.e.,

$$
d \eta^{\prime}=\left(1-\frac{m^{2}}{a^{2}}\right)^{-1 / 2} d \eta
$$

Inserting then (4.2) into (4.1), it is found that (modulo factor ordering)

$$
\left(-\frac{\partial^{2}}{\partial a^{2}}+\frac{\partial^{2}}{\partial \chi^{2}}+a^{2}-\chi^{2}\right) \Psi=m^{2} \Psi .
$$

This is the wave equation for wormholes or baby universes that we shall use in what follows. The LHS of (4.3) is identical to that in the conventional WheelerDeWitt equation as given in (3.3). It is the nonzero RHS of Eq. (4.3) that separates from the usual zero in Wheeler-DeWitt equation and corresponds to a total energy for the wormhole or little baby universes of the order the Planck energy. In Appendix $C$ we show that this wave equation is equivalent to a Klein-Gordon wave equation for wormholes.

The wave function $\Psi$ in (4.3) should now be interpreted as the quantum state of the wormhole or little baby universes. Since the analytical structure of the wormhole solution obtained from (4.1) is the same as that derived from (3.2), the use of (3.1) and (4.3) would lead to an effective interaction Lagrangian for flat space which is the same as (3.4). In fact, all the technical details involved in the derivation of the effective interactions in flat space between the branching off of little universes and low-energy particles are insensitive to variations of wormhole radius of the order the Planck length. At first glance it could seem unclear, however, whether a nonzero energy for wormholes would not affect the way through which a wormhole end is inserted in an infinitesimal volume of the manifold. Nevertheless, if one wants to respect general covariance and assumes, as is done in the derivation of (3.4) for zero-energy baby universes, ${ }^{31}$ that the amplitude for that insertion has the simplest form $C g^{1 / 2} d^{4} x$, the only effect originating from introducing a nonzero energy for baby universes is to induce a given finite change in the value of the constant $C$, so that the new $C^{\prime}$ still is a constant.

Actually, the value of the constant $C$ would be modulated by a factor $P \propto \epsilon^{-1}$ ( $\epsilon$ denoting total energy of the 
baby universes), interpretable as a probability for the wormhole state. The case for the Wheeler-DeWitt equation (4.3) would then correspond to the wormhole ground state, so that the probability factor $P$ could be set equal to unity. However, if we generalize to a full set of wormhole states with eigenenergies $\epsilon_{m}$, one should modify the insertion expression to read $\epsilon_{m}^{-1} C^{1 / 2} d^{4} x$. Unless otherwise stated, we shall restrict ourselves here to the simplest case represented by the Wheeler-DeWitt equation (4.3).

On the other hand, Eq. (4.3) is providing us with a natural mechanism for canceling the Planck zero-point energies of each scale factor harmonic-oscillator mode. Now these zero-point energies are simply canceled by the RHS term in Eq. (4.3). Thus, by using a parallel, though a more transparent procedure than Hawking's, ${ }^{5}$ we obtain again an effective Lagrangian for the interactions between wormholes and the background scalar matter fields in flat space of the form (3.4).

However, although the interaction equation should be formally the same as (3.5), its interpretation has to be necessarily different. Because the total energy of the baby universes is no longer zero, they, or at least their effects, could be observed by observers in the asymptotically flat region. This has two immediate consequences; first of all, one should exclude from the very beginning any possibility for the loss of quantum coherence induced by wormholes, and second, if one wants to respect energy conservation, the fundamental process branching off a baby universe from flat space has to be accompanied by loss or gain of an energy equal to that of baby universes by the matter field. The physical situation becomes so similar to that for the interaction between an electromagnetic field and a two-level matter system; or in other words, the study of the effects of wormholes on matter fields in asymptotically flat spaces is reducible to a quantum-optical problem.

In what follows we shall explore one of the typical consequences that can be expected from this analogy: the possibility for the spontaneous generation of chaotic behavior of spacetime induced by wormholes.

In the model we are dealing with, this possibility is a very plausible one; after all, the branching off of little baby universes from a large universe has the following characteristics: It is governed by nonlinear equations, it can induce an energy feedback mechanism between large universes, and it converts an otherwise closed large universe into a dissipative system. These are precisely the basic requirements for a system to have the possibility of showing chaotic behavior.

It could be thought that, since wormholes are events at Euclidean spacetime, it would be meaningless to attempt deriving a dynamical equation of the wormhole gas. For this dynamics to have any physical meaning, one should require having (i) a suitable well-defined time variable to describe the interaction, and, (ii) in the case of a Euclidean spacetime manifold, some mechanism by which the Euclidean dynamical equation would manifest in some observable effect in the asymptotic or Lorentzian region. For the interaction being considered, the first of these two conditions is satisfied as the existence of a nonzero total energy term in (4.3) allows us to obtain a full wave equation (see Appendix C), where the energy term can be generated by introducing a time operator $\partial / \partial t$, much in the same way as it is done in nongravitational quantum mechanics. Having such a time operator makes it possible to introduce consistent operator ordering and a Hilbert space for the wormhole states where one can consistently define unitary time-evolution operators or Heisenberg equations of motion.

On the other hand, it is known that wormholes may induce observable effects on matter in the asymptotic region through an effective Lagrangian with the general form (3.4). Clearly, one should likewise expect that the dynamics of a gas of wormholes or baby universes derived from the same effective interaction Lagrangian plus an independent Lagrangian for the baby universes and matter fields would also induce observable effects in the asymptotic region. Any effect that such wormhole dynamics may produce in the structure of the Euclidean spacetime itself would be observable not just in the asymptotic region, but also in the Lorentzian interior of the little universes that are branched off from such an asymptotic region. The possibility that we are living in a universe which was once born as one of such baby universes creates some additional interest in the study of the dynamical equation of the wormhole gas.

The general configuration we shall consider consists of two large three-spaces $M_{1}$ and $M_{2}$ and a given number $n$ of observable baby universes which connect the two large spaces. $M_{1}$ and $M_{2}$ are themselves connected by a fourmanifold $M$. Then, if $\phi$ denotes the background scalar matter fields, and $b_{u}$ denotes the field of baby universes, we have an effective Lagrange density of the form

$$
L=L_{m}(\phi)+L_{w}\left(b_{u}\right)+\sum_{i} L_{i}(\phi)\left(b_{i}^{\dagger}+b_{i}\right),
$$

where $L_{m}$ and $L_{i}$ are local functions of the background fields and their derivatives, and $L_{w}$ is a new Lagrange density term accounting for free baby universes.

Assuming only one kind of baby universe and only one kind of background field, the corresponding Hamiltonian for the total system can be written in the typical quantum-optical form

$$
H=\sum_{j}\left[\omega_{w} b^{\dagger} b+\frac{1}{2} \omega_{\phi} \rho_{j}+i \omega_{P}\left(\sigma_{j}^{-} b^{\dagger}+\sigma_{j}^{+} b\right)\right],
$$

where $\omega_{w}$ denotes the wormhole energy (i.e., the inverse of the wormhole radius in the case of pure gravity), $\omega_{\phi}$ is the energy shift produced in the matter field in flat space by the interaction (typically, this would be of the order the Planck scale), and $\omega_{P}$ is of the order of Planck energy. The index $j$ accounts for the local matter systems.

As pointed out before, $b^{\dagger}, b$ are Fock operators for creation and annihilation of baby universes, satisfying the usual commutation relations for bosons:

$$
\begin{aligned}
& {\left[b^{\dagger}, b\right]=1,} \\
& {\left[b^{\dagger}, b^{\dagger}\right]=[b, b]=0 .}
\end{aligned}
$$

In writing Eq. (4.5) it has been assumed, furthermore, that the matter fields may absorb or emit the energy of a 
baby universe, and therefore these fields behave as if they were typical two-level systems. Note that, whereas in the conformally invariant field model $\Phi \neq 0$ the energy of the ground state would correspond to the bare energy of the background field, in the more general nonfield model $\Phi=0$, the ground-state energy would be zero. Consequently, we have introduced the isospin transition operators $\sigma_{j}^{ \pm}$which raise $(+)$or lower $(-)$the energy level of the background matter system. Operators $\rho_{j}$ fix the total energy of the matter fields. All of these isospin operators satisfy the commutation relations

$$
\begin{aligned}
& {\left[\sigma_{j}^{+}, \sigma_{j}^{-}\right]=\rho_{j},} \\
& {\left[\rho_{j}, \sigma_{j}^{ \pm}\right]= \pm 2 \sigma_{j}^{ \pm} .}
\end{aligned}
$$

Using then the Heisenberg equations of motion, we obtain

$$
\begin{aligned}
& \dot{b}+i \omega_{w} b=\omega_{P} \sum_{j} \sigma_{j}^{-}, \\
& \sum_{j}\left(\dot{\sigma}_{j}^{-}+i \omega_{\phi} \sigma_{j}^{-}\right)=\omega_{P} \sum_{j} \rho_{j}, \\
& \sum_{j} \dot{\rho}_{j}=-2 \omega_{P} \sum_{j}\left(b \sigma_{j}^{+}+b^{\dagger} \sigma_{j}^{-}\right) .
\end{aligned}
$$

Equation (4.4), (4.5), and (4.8) are given in first approximation. The real interaction process would not be so simple because of a variety of effects, including interactions of the background field with the surrounding medium, interactions among wormholes, the distribution of the most probable values of the physical constants in large universes according to the Coleman mechanism, ${ }^{10}$ etc. On the other hand, in the more realistic quantum approach using a density matrix to describe the quantum state of a single wormhole, we had to sum over all the possible wormhole insertion amplitudes weighted by probability factors depending on the inverse of the wormhole eigenenergies. These probabilities would also influence the average values of the relevant dynamical parameters appearing in the resulting equations of motion. For example, let us consider what would happen if the dilute wormhole approximation breaks down (a situation that turns out to be very likely as one is dealing with smaller universes). In this case we would have to introduce processes such as two wormholes being branched off close to one another or the equivalent process by which two wormholes coalesce to form a third one, which is then absorbed, etc. All of these processes and interactions would require a given careful rebalancing of the energetics of the whole process, which can be generally accounted for by adding phenomenological coupling constants to the different operators in Eq. (4.8). If we denote these constants by $\gamma_{b}, \gamma_{\sigma}$, and $\gamma_{\rho}$, we can generalize the above set of equations to read

$$
\begin{aligned}
& \dot{b}+\left(i \omega_{w}+\gamma_{b}\right) b=\omega_{P} \sum_{j} \sigma_{j}^{-}, \\
& \sum_{j}\left[\dot{\sigma}_{j}^{-}+\left(i \omega_{\phi}+\gamma_{\sigma_{j}^{-}}\right) \sigma_{j}^{-}\right]=\omega_{P} \sum_{j} \rho_{j}, \\
& \sum_{j}\left(\dot{\rho}_{j}+\gamma_{\rho_{j}} \rho_{j}\right)=-2 \omega_{P} \sum_{j}\left(b \sigma_{j}^{+}-b^{\dagger} \sigma_{j}^{-}\right) .
\end{aligned}
$$

If we consider now that the coupling constants do not depend on the index $j$, use the approximation $\left\langle b \sigma_{j}^{ \pm}\right\rangle=\langle b\rangle\left\langle\sigma_{j}^{ \pm}\right\rangle$, etc., and change the notation so that $b \rightarrow W, \Sigma_{j} \sigma_{j}^{-} \rightarrow A, \Sigma_{j} \sigma_{j}^{+} \rightarrow C$, and $\Sigma_{j} \rho_{j} \rightarrow M$, Eqs. (4.9) can be rewritten as

$$
\begin{aligned}
& \dot{\boldsymbol{W}}+\left(i \omega_{w}+\gamma_{w}\right) \boldsymbol{W}=\omega_{P} \boldsymbol{A}, \\
& \dot{A}+\left(i \omega_{\phi}+\gamma_{A}\right) \boldsymbol{A}=\omega_{P} \boldsymbol{M}, \\
& \dot{\boldsymbol{M}}+\gamma_{M} \boldsymbol{M}=-2 \omega_{P}\left(\boldsymbol{W C}-\boldsymbol{W}^{*} \boldsymbol{A}\right) .
\end{aligned}
$$

The derivation of Eqs. (4.10) given here is rather heuristic; it is expected that a more formal derivation would leave untouched the general form of these equations. Note that for sufficiently high density of wormholes, one could even have stimulated emission processes induced by the baby universes. The point now is to realize that the set of Eq. (4.10) may be derived equivalent to the Lorenz equations ${ }^{32}$ describing the behavior of systems which can deterministically evolve to chaos. It could well be then that for given values of the parameters involved in Eqs. (4.10), the branching off of little baby universes from asymptotically flat regions of space would lead to chaotic behavior of spacetime itself. Such a behavior could be expected at some epoch of the early Universe, when the combined effect of the smallness of the Universe with suitably high values of the control parameters would allow chaos to pervade the whole spacetime structure of the Universe. Thus such an Euclidean chaos would probably take place in an early stage of the evolution of the Universe, at which time, at least according to the "no-boundary" proposal, the Universe was Euclidean and expanded exponentially.

\section{VANISHING OF THE COSMOLOGICAL CONSTANT AND THE BIG FIX}

In this section we shall briefly review some of the possible implications that the quantum-optical interpretation of wormhole interactions may have in the Coleman mechanism ${ }^{10,31}$ for the vanishing of the cosmological constant and the fixing of the physical coupling constants.

Coleman's mechanism is based on the result that, because of interaction with wormholes, any expectation value computed in one large universe is a weighted average over expectation values in an arbitrarily large number of universes without wormholes, but with wormholeshifted couplings $\lambda+\alpha$, where $\lambda$ collectively denotes parameters such as the gravitational constant, fundamental particle masses, or the cosmological constant; $\alpha$ denotes a set of fixing parameters defined by the identity

$$
\begin{array}{rl}
\exp \left(\frac{1}{2} C_{i j} \phi_{i} \phi_{j}\right) \sim \int \prod_{k} & d \alpha_{k} \exp \left(-\frac{1}{2} D_{i j} \alpha_{i} \alpha_{j}\right) \\
& \times \exp \left(-\alpha_{l} \phi_{l}\right),
\end{array}
$$

in which $C_{i j} \sim \exp \left(-I_{w}\right), \phi(x)$ is the basis for a local operator at $x, D_{i j}$ is the inverse of matrix $C_{i j}$, and $I_{w}$ is the wormhole action.

In the dilute wormhole approximation, taking the classical stationary point associated with Euclidean de Sitter 
space as the leading contribution to the Euclidean path integral

$$
\int d g \exp [-I(g, \lambda+\alpha)]
$$

over smooth metrics and all other fields in one large universe, Coleman was able to show ${ }^{10}$ that the probability function for an ensemble of worlds with a statistical distribution of coupling constants is given by

$$
\begin{aligned}
P \sim \exp & \left(-\frac{1}{2} D_{i j} \alpha_{i} \alpha_{j}\right) \exp \left(\frac{3}{8 G^{2} \Lambda}-\frac{8 \pi^{2}}{3} A_{1}\right) \\
& \times \exp \left[\exp \left(\frac{3}{8 G^{2} \Lambda}-\frac{8 \pi^{2}}{3} A_{1}\right)\right],
\end{aligned}
$$

where $\Lambda \equiv \Lambda(\alpha)$ and $G \equiv G(\lambda)$ denote, respectively, physical values of the cosmological and gravitational constants, and $A_{1} \equiv A_{1}(\alpha)$ is the coefficient for the firstorder field fluctuation in the effective action for a large smooth universe.

The analysis of the probability function $P$ in terms of the first term $3 / 8 G^{2} \Lambda$ of the argument of the single and double exponentials is the basis for the Coleman's claim for a wormhole-induced vanishing of the cosmological constant $\Lambda$. Since $P$ is infinitely peaked at $\Lambda=0$, zero is by far the most probable physical value for the cosmological constant. ${ }^{15}$

It seems clear that the Coleman analysis for the vanishing of the cosmological constant does not depend on the details of physics at around the Planck scale ${ }^{31}$ and therefore it remains valid in the quantum-optical interpretation of wormhole interactions with large universes. This is a very plausible conclusion as $\Lambda$ is a cosmological parameter which ought to be sensitive only to the largescale characteristics of the considered universe. However, if the large universe is an expanding system, the above conclusion would no longer be valid in the earliest stages of its evolution when the scale factor is comparable with the Planck length. Thus details of small-distance physics, such as those discussed in Secs. II and IV, would probably affect expression (5.2), or at least its interpretation, in such a way that the resulting probability would be peaked at some nonzero value of the cosmological constant.

The Coleman argument for the so-called big fix refers to the second term $-8 \pi^{2} / 3 A_{1}$ and, potentially, higher perturbations terms, in the argument of the single and double exponentials in (5.2). The absolute probability maximum at $G^{2} \Lambda(\alpha)=0$ defines some subspace in the space of wormhole-shifted constants where the probability varies and becomes infinitely sharply peaked at the place where $A_{1}(\alpha)$ reaches its minimum. In this case, however, power counting indicates ${ }^{31}$ that $A_{1}(\alpha)$ and the other coefficients of the expansion depend strongly on short-distance physics for which the usual and quantumoptical interpretations of wormhole interactions are remarkably different. Therefore, the introduction of a universal cutoff of order $m$ in the Coleman scenario ought to lead to a different interpretation of the perturbation coefficients.

To get a more precise idea about how the probability
$P(\alpha)$ would separate from the Coleman formula when one introduces an extra uncertainty in wormhole physics, let us generalize the formalism to the single-wormholestate density matrix representation. For a wormhole interacting with a single scalar field, the bilocal effective interaction is proportional to

$$
-\frac{1}{2} \sum_{i j} C_{i j}^{m} \beta^{2 m} \epsilon_{m}^{-1}
$$

where $m$ labels the energy levels of the wormhole harmonic-oscillator states, $m=1,2,3, \ldots, \quad$ and $\beta^{2}=\frac{1}{2}\left[\int d^{4} x g(x)^{1 / 2} V\right]^{2}$, with $V$ being a vertex operator, and $\epsilon_{m} \propto m$. The minus sign in (5.3) is introduced according to the discussion by Hawking. ${ }^{31}$

We should now sum over all the states $m$. Neglecting the wormhole-wormhole interactions, the sum of (5.3) will exponentiate already for a single wormhole. If we next sum over any number of wormholes, each having a similar harmonic-oscillator spectrum, connecting a single large universe characterized by an Euclidean action $I(g, \lambda)$, then one has a still bilocal probability proportional to

$$
e^{-I(g, \lambda)} \exp \left[-\int d \beta \beta^{-1} \exp \left(\sum_{i j} C_{i j} \beta^{2}\right)\right] .
$$

Now, by using the integral

$$
e^{-b^{2} / 4 a}=\left(-\frac{a}{\pi}\right)^{1 / 2} \int_{-\infty}^{+\infty} d \alpha e^{a \alpha^{2}} e^{b \alpha},
$$

(5.4) can be made to look local. We get

$\exp \left\{-\int d \alpha\left[I(g, \lambda)-\left(\frac{D_{i j}}{4 \pi}\right)^{1 / 2} e^{-D_{i j} \alpha^{2} / 4} E_{1}(\alpha \beta)\right]\right\}$,

where $E_{1}(x)$ is the exponential integral of first order.

For the sake of simplicity, let us now consider the case in which we set large values for the product $\alpha \beta$. Similar results may be obtained for any value of $\alpha \beta$. Assuming processes involving additional large universes, one would finally obtain a probability given by

$$
\begin{aligned}
\exp \left\{\exp \left[-\int d \alpha I\left[g, \lambda_{n}+\frac{D^{1 / 2} e^{-D \alpha^{2} / 4} \alpha^{n}}{f(n)}\right]\right]\right\} & \\
& \times \exp \left(-\int d \alpha D^{1 / 2} e^{-D \alpha^{2} / 4}\right],
\end{aligned}
$$

where $n$ labels the coefficients for the power expansion of $E_{1}$ and $f(n)$ are the associated numerical coefficients.

Again, the first exponential factor in (5.6) dominates over the second one, and one can still predict zero as by far the most probable value for the cosmological constant. However, expression (5.6) shows a crucial difference with respect to its Coleman counterpart. Now, depending on the sign of the term being considered, parameters $\alpha$ cannot take on any unboundedly either large or small value. The shifts affecting the couplings do always possess a finite extremum in $\alpha$. If the sign is positive, then the extremum will correspond to a finite maximum at 


$$
\alpha_{0}=\left(2 n e^{-S_{w}}\right)^{1 / 2} ;
$$

if the sign is negative, the extremum will be a finite minimum at the same value (5.7) of $\alpha$. This obviously restricts the range of possible values for the Coleman parameters and, thereby, may drastically change the most probable values of the coupling constants predicted by Coleman model.

Let us consider, for example, the typical case of a light pseudoscalar particle, such as the pion. Assuming that the pion mass $m_{\pi}$ is much smaller than the large QCD scale $f_{\pi}$, Grinstein and Wise ${ }^{33}$ have obtained the coefficient $A_{1}$ for this case:

$$
A_{1}=B \ln \left(\frac{m_{\pi}^{2}}{f_{\pi}^{2}}\right)+O\left(\frac{m_{\pi}^{2}}{f_{\pi}^{2}}\right),
$$

where $B$ is a positive numerical coefficient of order unity.

Thus $P$ becomes infinitely sharply peaked at $m_{\pi}=0$, a result which is certainly very discouraging. Nevertheless, let us look at what would actually happen when, in the close vicinity of a pion in flat space, little baby universes are being branched off. Of course, the bare mass of the pion will get a dependence on parameters $\alpha$ such as that discovered by Coleman; but if one adopts the quantumoptical interpretation of wormhole interactions, that dependence will not be the sole memory left by wormholes in the mass of pions. In creating a wormhole with total energy $m^{-1}$, for example, the bare mass of the pion would change to $\hat{m}_{\pi} \sim m_{\pi}-m^{-1}+\gamma_{M}$, where $\gamma_{M}$ is the phenomenological coupling constant for operator $\rho$ introduced in Sec. IV. Hence the dependence of Eq. (5.8) on the pion mass would now be through $\hat{m}_{\pi}$ instead of $m_{\pi}$, and since the probability would still be infinitely sharply peaked at $\hat{m}_{\pi}=0$, the physical pion mass should no longer be driven to zero, but to a generally nonzero value

$$
m_{\pi} \sim m^{-1}(\alpha)-\gamma_{M}(\alpha) .
$$

Similar arguments should apply to fermions, where the numerical coefficient turns out to be negative, ${ }^{31,34}$ so that wormholes would ultimately drive the free-fermion mass toward $\Pi-m^{-1}(\alpha)+\gamma_{M}(\alpha)$, with II being the cutoff scale of the theory. Thus phenomenological coupling constants, such as $\gamma_{M}(\alpha)$, would ultimately fix the value of the masses of the fundamental particles. As discussed in Sec. IV, the values of these constants are expected to depend, among other things, on nonlinear wormhole interactions and on the size of the universe. Therefore, although the big-fix program could be saved by using nonzero energy wormholes, it seems that the day when there will be a consistent theory for the determination of every physical constant is still discouragingly far away.

\section{CONCLUSIONS AND FURTHER COMMENTS}

The available classical wormhole solutions obtained as instantons from Euclidean gravity coupled to special kinds of matter fields have all a restricted applicability and, at least in one case, show a severe problem with the value of the effective gravitational constant. Therefore, they cannot be invoked as a solution to problems so universal as that of the cosmological constant or the fixing of physical coupling constants.

Starting with pure gravity or a scalar field coupled to gravity, we have used a new general method to obtain the Tolman-Hawking wormhole instanton by simply providing the Robertson-Walker manifold with an extra threesphere of minimum radius of about the Planck length. In either of these two cases, there is no problem with the value of the effective gravitational constant, provided that we introduce a cutoff in the wormhole scale factor squared at the Planck scale. It was found that the wave equation for this kind of solution is no longer equal to zero, as it is in the conventional Wheeler-DeWitt equation, but to a nonzero energy of the order the Planck energy. Therefore, the little baby universes resulting at the cross sections of wormholes have a nonzero total energy. Baby universes would then be observable and induce no loss of quantum coherence whatsoever.

By using an analysis in terms of second-order correlation functions, we have also shown that, even for little closed universes, there could be no loss of quantum coherence induced by quantum gravity.

By invoking energy conservation, it was suggested that the interaction between low-energy particles in asymptotically flat spaces and wormholes can be reduced to a typical process of quantum optics. A striking consequence from this interpretation is the possibility discussed in this paper that wormholes may induce chaotic behavior of spacetime itself. Because of the nonzero RHS term of the wave equation governing the evolution of the quantum state of wormholes, analogously to what is done in the nongravitational picture of ordinary quantum mechanics, one could introduce a well-defined notion of time in quantum gravity (see Appendix C) that allowed the suggested gravitational chaos to be deterministically accounted for. It is expected, moreover, that the longstanding operator-ordering problem ${ }^{35}$ of canonical quantum gravity may find a natural solution within the context of this picture.

Chaotic spacetime could then be interpreted as the most natural initial condition for our Universe. Actually, the quantum-optical interpretation of wormhole-matter interaction could thus implement the unique inflationary model that has survived so far, i.e., the chaotic inflationary scenario proposed by Linde. ${ }^{36}$ If so, the problem of the initial conditions of the Universe could be reduced to find a suitable set of parameters that would induce the chaotic behavior of spacetime, starting from an initial soup of wormholes.

Our interpretation of wormhole interaction is compatible with the Coleman mechanism for the vanishing of the cosmological constant in large universes, but not with the big-fix program as formulated by Coleman. It appears that our interpretation could drastically improve the discouraging results obtained so far.

In Appendix $\mathrm{C}$ it is shown that the Wheeler-DeWitt equation for wormholes is equivalent to a Klein-Gordon equation. Hence the wave function representing the quantum state of wormholes or little baby universes would admit a probabilistic interpretation equivalent to 
the Copenhagen interpretation of relativistic quantum mechanics. The concept of antiwormholes, i.e., nontrivial topological changes carrying negative energy, is introduced without violating the Ruback uniqueness theorem for wormholes.

In summary, one could answer the question posed in the title of this paper by saying that it could well be that nothing is actually being lost in wormholes; quite the opposite, it appears that one may gain a lot from them. Apart from getting rid of the cosmological constant and allowing the possibility of dynamically generating all physical coupling constants, wormholes may be providing us with suitable mechanisms for inducing deterministic chaos in spacetime and generating all known particles themselves as nontrivial gravitational instantons; after all, it seems that the little baby universes branching off from flat space carry nonzero energy and obey the fundamental laws of nongravitational relativistic quantum mechanics.

\section{ACKNOWLEDGMENTS}

The author is grateful to $\mathbf{R}$. Laflamme for very enlightening discussions on Euclidean path-integral formulation of wormholes and the meaning of the loss of quantum coherence in wormhole physics. It is also a pleasure to thank R. Harrison for discussion on deterministic chaos in quantum-optical systems. Conversations with S. W. Hawking, J. A. Torresano, G. Mena Marugan, L. Garay Elizondo, L. M. Campbell, and J. Perez-Mercader are also gratefully acknowledged. I finally thank support in part by the Spain-Great Britain Cooperative Project No. $58 / 146$.

\section{APPENDIX A: LORENTZIAN SOLUTIONS FOR A TOLMAN-HAWKING WORMHOLE}

In the Lorentzian region we would have a transformed Robertson-Walker time

$$
d t^{\prime}=\left[\frac{m^{2}}{a^{2}}-1\right]^{1 / 2} d t
$$

hence the Lorentzian region is defined for $a<m$.

By using the Lorentzian field equations obtained from their Euclidean counterparts (2.13), introducing the usual change $\eta \rightarrow i \eta$, we get the wanted solutions. For $\lambda=0$,

$$
a=\left(M^{2}+m^{2}-t^{2}\right)^{1 / 2},
$$

which is expressed in terms of Robertson-Walker time $t$ and would correspond to a Tolman universe. ${ }^{17}$

In this case, however, the presence of a scalar field conformally coupled to gravity can avoid the Lorentzian singularities at $t_{s}= \pm\left(M^{2}+m^{2}\right)^{1 / 2}$ because the Lorentzian regime requires $t^{2}>M^{2}$. In any event, the maximum radius of the Tolman universe appears at $t=0$, with $a_{\max }=\left(M^{2}+m^{2}\right)^{1 / 2}$.

The only possible Lorentzian solution for $\lambda>0$ is

$$
\begin{gathered}
a(t)=(2 \lambda)^{-1 / 2}\left[1+2 m^{2} \lambda-\left(1-4 \lambda M^{2}\right)^{1 / 2}\right. \\
\left.\times \cosh \left(2 \lambda^{1 / 2} t\right)\right]^{1 / 2},
\end{gathered}
$$

which is defined for $4 \lambda M^{2}<1$ and $0<a<a_{-}$.

Solution (A3) is also a Tolman-like Lorentzian solution, only at times defined by $t>t_{c}$, where

$$
t_{c}=\left(2 \lambda^{1 / 2}\right)^{-1} \operatorname{arccosh}\left[\left(1-4 \lambda M^{2}\right)^{-1 / 2}\right] .
$$

In principle, there are two more solutions to the Lorentzian equations of motion. They are

$$
\begin{gathered}
a(t)=(2 \lambda)^{-1 / 2}\left[1+2 m^{2} \lambda+\left(1-4 \lambda M^{2}\right)^{1 / 2}\right. \\
\left.\times \cosh \left(2 \lambda^{1 / 2} t\right)\right]^{1 / 2},
\end{gathered}
$$

for $a>a_{+}$and $4 \lambda M^{2}<1$, and

$$
\begin{gathered}
a(t)=(2 \lambda)^{-1 / 2}\left[1+2 m^{2} \lambda+\left(4 \lambda M^{2}-1\right)^{1 / 2}\right. \\
\left.\times \sinh \left(2 \lambda^{1 / 2} t\right)\right]^{1 / 2},
\end{gathered}
$$

for $4 \lambda M^{2}>1$. However, such de Sitter-like solutions are defined for $a>m$ and, therefore, are not allowed in the Lorentzian regime.

\section{APPENDIX B: TRANSFORMED CONFORMAL TIME}

In this Appendix we derive first the relation between $\eta$ and $\eta^{\prime}$ and discuss then in more detail why the correspondence principle introducing the quantum operator $\partial / \partial \chi$ should be applied to $d \chi / d \eta^{\prime}$ instead of $d \chi / d \eta$.

From the relation [cf. Eq. (4.2)]

$$
\eta^{\prime}=\int\left(1-\frac{m^{2}}{a^{2}}\right)^{-1 / 2} d \eta
$$

we get

$$
\eta^{\prime}=\ln \left[\sinh \eta+\left(\frac{M^{2}}{m^{2}+M^{2}}+\sinh ^{2} \eta\right]^{1 / 2}\right] .
$$

We see that $\eta^{\prime}$ covers only half the $\eta$ interval, from $\eta=+\infty$ to 0 . For a given nonzero field $\chi$, the time $\eta^{\prime}$ runs first from $+\infty$ (at $\eta=+\infty$ ) to 0 (at $\left.\eta=\operatorname{arcsinh}\left[m^{2} / 2\left(m^{2}+M^{2}\right)\right]\right)$ and then to $+\ln \left[M /\left(m^{2}+M^{2}\right)^{1 / 2}\right]($ at $\eta=0)$. It is only for the case $M=0$ that $\eta^{\prime}$ runs the entire range up to $-\infty$. In this case, $\eta^{\prime}$ reduces to

$$
\eta^{\prime}=\ln 2+\ln (\sinh \eta) \text {. }
$$

Let us now investigate which conformal time derivative of the field $\chi$ should correspond to the field quantum momentum operator. We note first the relations

$$
\begin{aligned}
& a^{\prime}=-i \chi=\left(a^{2}-m^{2}-M^{2}\right)^{1 / 2}, \\
& \chi^{\prime}=i a=\left(\chi^{2}-m^{2}-M^{2}\right)^{1 / 2} .
\end{aligned}
$$

Now, if we choose a correspondence principle $a^{\prime} \Longrightarrow \partial / \partial a$ for the scale factor, Eqs. (B4 and B5) indicate that, since we have not explicitly shifted the scalar field, $\chi^{\prime}$ could no longer be the relevant field quantity to be associated with the operator $\partial / \partial \chi$, for Eq. (B5) tells us that $\chi^{\prime}$ contains the unwanted shift in $m^{2}$. Nevertheless, the quantity

$$
\frac{d \chi}{d \eta^{\prime}}=i\left(a^{2}-m^{2}\right)^{1 / 2}=\left(\chi^{2}-M^{2}\right)^{1 / 2}
$$

satisfies all the requirements and becomes; therefore, the suitable field quantity to be associated with the quantum operator $\partial / \partial \chi$.

This can be also seen by using a more transparent argument. In the gauge $N=1$, we obtain from (2.12) the Lagrangian 


$$
\begin{aligned}
L=L(\eta) & =\frac{1}{2}\left(a^{2}-m^{2}\right)^{1 / 2}\left[-\frac{a^{\prime 2}}{a^{2}-m^{2}}-1+\lambda\left(a^{2}-m^{2}\right)+\frac{\chi^{\prime 2}}{a^{2}}+\frac{\chi^{2}}{a^{2}-m^{2}}\right] \\
& =L_{a}(\eta)+L_{\chi}\left(\eta^{\prime}\right)=\frac{1}{2}\left(a^{2}-m^{2}\right)^{1 / 2}\left(-\frac{a^{\prime 2}}{a^{2}-m^{2}}-1+\lambda\left(a^{2}-m^{2}\right)\right]+\frac{1}{2}\left(a^{2}-m^{2}\right)^{1 / 2}\left(\frac{\left(d \chi / d \eta^{\prime}\right)^{2}}{a^{2}-m^{2}}+\frac{\chi^{2}}{a^{2}-m^{2}}\right) .
\end{aligned}
$$

From this Lagrangian, we obtain the momenta for the scale factor and scalar field:

$$
\begin{aligned}
& p_{a}=\frac{\partial L(\eta)}{\partial a^{\prime}}=\frac{\partial L_{a}(\eta)}{\partial a^{\prime}}=-\frac{a^{\prime}}{\left(a^{2}-m^{2}\right)^{1 / 2}}, \\
& p_{\chi}=\frac{\partial L(\eta)}{\partial \chi^{\prime}}=\frac{\left(a^{2}-m^{2}\right)^{1 / 2} \chi^{\prime}}{a^{2}}, \\
& p_{\chi}^{\prime}=\frac{\partial L_{\chi}\left(\eta^{\prime}\right)}{\partial\left(d \chi / d \eta^{\prime}\right)}=\frac{d \chi / d \eta^{\prime}}{\left(a^{2}-m^{2}\right)^{1 / 2}} .
\end{aligned}
$$

Now, because the field momentum which is formally equivalent to $p_{a}$ is not $p_{\chi}$, but $p_{\chi}^{\prime}$, it follows that if we associate the quantum operator $\partial / \partial a$ with $a^{\prime}$, the field quantity to be equivalently associated with the operator $\partial / \partial \chi$ is $d \chi / d \eta^{\prime}$.

\section{APPENDIX C: QUANTUM MECHANICS OF WORMHOLES}

In this Appendix we derive a Klein-Gordon wave equation for wormholes and comment briefly on it. We start with Eq. (4.3):

$$
\left(-\frac{\partial^{2}}{\partial a^{2}}+\frac{\partial^{2}}{\partial \chi^{2}}+a^{2}-\chi^{2}\right) \psi=m^{2} \psi .
$$

It will first be noted that for an integration constant $M=0$ we still have a residual scalar field of the form

$$
\chi_{M=0} \equiv \Xi=i m \sinh \eta
$$

which suggests that the introduction of a maximum resolution limit of the form $\Delta a \geq m$ is equivalent to having a background purely imaginary quantum field $\Xi$ which only vanishes at the classical limit $m \rightarrow 0$ or at $\eta=0$. In other words, if a Tolman-Hawking wormhole exists, there must exist an axionlike imaginary scalar field so that $a^{\prime 2}+\chi^{2}=0, \chi^{\prime 2}+a^{2}=0$. The field $\Phi=m_{P} \tanh \eta$ is zero at $\eta=0$, and $\pm i m_{P}$ at the asymptotic region $\eta= \pm \infty$.

We note then that one can obtain a relation between the Euclidean Robertson-Walker and conformal times from Eqs. (2.15) and (2.19) of the form

$$
\tau=\int a d \eta=\left(m^{2}+M^{2}\right)^{1 / 2} \sinh \eta=-i \chi .
$$

This is an interesting result which tells us that the conformally coupled scalar field $\chi$ is nothing but the Lorentzian Robertson-Walker time $t$. Equivalently, the quantum scalar field $\Xi$ will define an intrinsic quantum Lorentzian Robertson-Walker time that we shall denote as $\Gamma$ and an intrinsic quantum Euclidean Robertson-
Walker time which will be denoted by $\Upsilon$, so that $\Gamma=i \Upsilon$. Then the modified Wheeler-DeWitt equation $(\mathrm{C} 1)$ can be rewritten as a true Euclidean Klein-Gordon wave equation describing the evolution of wormhole states:

$$
\left[\frac{\partial^{2}}{\partial a^{2}}+\frac{\partial^{2}}{\partial \tau^{2}}+V(\chi, m)-\left(m^{2}+M^{2}\right)\right] \psi=0,
$$

where the potential $V(\chi, m)$ is given by

$$
V(\chi, m)=2 \chi^{2}+m^{2},
$$

and the wave function that describes the quantum state of the wormhole or little baby universes,

$$
\psi \equiv \psi(a, \chi) \equiv \psi(a, \tau),
$$

will depend on Euclidean Robertson-Walker time.

For pure gravity, (C4) becomes

$$
\left(\frac{\partial^{2}}{\partial a^{2}}+\frac{\partial^{2}}{\partial \Upsilon^{2}}+U(\Xi)-m^{2}\right) \Phi=0,
$$

where

$$
U(\Xi)=2 \Xi^{2},
$$

and

$$
\Phi \equiv \Phi(a, \Xi) \equiv \Phi(a, \Upsilon)
$$

The time dependence of the wormhole quantum state is not surprising because the total energy of the wormhole is nonzero. From this result a number of interesting consequences can be expected. Among them, we remark here the following two: (i) A self-consistent factor ordering for canonical quantum gravity could now be established, and (ii) there exists the possibility of deriving linearized wave equations from (C4) or (C7) by suitably introducing Pauli matrices. This would lead to a given spin for wormholes and to the notion of antiwormholes, i.e., topological changes carrying negative energy.

Working in pure gravity, we have, for the Lorentzian version of (C7),

$$
\left[\hat{E}^{2}-\hat{p}_{a}^{2}-2 \Gamma^{2}-(i m)^{2}\right] \Phi_{L}=0,
$$

where $\widehat{E}=i m \partial / \partial \Gamma, \hat{p}_{a}=i m \partial / \partial a$, and $\Phi_{L}$ represents the quantum state of a Tolman universe with the Planck size. Since we are considering an isotropic manifold, we may obtain a Dirac wave equation for the Tolman universe of the form

$$
\left[\widehat{E}-\sigma_{1} \hat{p}_{a}-2^{1 / 2} \sigma_{3} \Gamma-i \sigma_{2} m\right] \Psi_{L}=0,
$$

with the $\sigma_{i}$ 's being the Pauli matrices and $\Psi_{L}$ being a two-component wave function. 
The Euclidean version of (C11) could then be interpreted as the Dirac wave equation for wormholes, i.e.,

$$
\left[-i \hat{\epsilon}-\sigma_{1} \hat{p}_{a}-2^{1 / 2} i \sigma_{3} \Upsilon-i \sigma_{2} m\right] \Psi_{w}=0 .
$$

In Eq. (C12), $\hat{\epsilon}=i m \partial / \partial \Upsilon$, and $\Psi_{w}$ is a two-component wave function denoting the quantum state of wormholes. Taking the complex conjugate of $(\mathrm{C} 12)$, it is readily seen that antiwormholes ought to be interpreted as negativeenergy wormholes or, alternatively, as wormholes spacetime acted upon by parity- and time-inversion operators $P: a \rightarrow-a$ and $\Upsilon \rightarrow-\Upsilon$.

The existence of an antiwormhole sector of the wave function implements the bivalued character of the Euclidean action arising from inserting the cutoff on the scale factor. In order to preserve convergence, one should use the Gibbons-Hawking-Perry prescription. ${ }^{37}$ Taking $N$ and $a$ imaginary, as it would correspond to integrating the conformal factor over a contour parallel to the imaginary axis, the Euclidean action for pure gravity becomes

$$
\widetilde{I}^{R}= \pm \int d \tau\left(a^{2}+m^{2}\right)^{1 / 2} N\left(\frac{a^{2} \dot{a}^{2}}{\left(a^{2}+m^{2}\right) N^{2}}+1\right)
$$

with $\tau \rightarrow \Upsilon$.

(C13)

The overall plus sign leaves a positive-definite action integral that should be associated with the wormhole sector. The minus sign should then correspond to the antiwormhole sector and would also give rise to a positivedefinite action as one applies $P$ and $T$ operators to it.
${ }^{1}$ See, e.g., S. W. Hawking, in General Relativity, An Einstein Centenary Survey, edited by S. W. Hawking and W. Israel (Cambridge University Press, Cambridge, England, 1979).

${ }^{2}$ A. D. Linde, Phys. Lett. B 200, 272 (1988).

${ }^{3}$ A. Strominger, presented at the TASI Summer School, Providence, Rhode Island, 1989 (unpublished).

${ }^{4}$ S. W. Hawking, Phys. Lett. B 195, 337 (1987).

${ }^{5}$ S. W. Hawking, Phys. Rev. D 37, 904 (1988).

${ }^{6}$ S. B. Giddings and A. Strominger, Nucl. Phys. B306, 890 (1988).

${ }^{7}$ G. V. Lavrelashvili, V. A. Rubakov, and P. G. Tinyakov, Pis'ma Zh. Eksp. Teor. Fiz. 46, 134 (1987) [JETP Lett. 46, 167 (1987)].

${ }^{8}$ S. Coleman, Nucl. Phys. B307, 867 (1988).

${ }^{9}$ S. B. Giddings and A. Strominger, Nucl. Phys. B307, 854 (1988).

${ }^{10}$ S. Coleman, Nucl. Phys. B310, 643 (1988).

${ }^{11}$ S. W. Hawking, Commun. Math. Phys. 87, 395 (1982).

12D. J. Gross, Nucl. Phys. B238, 349 (1984).

${ }^{13}$ S. W. Hawking and R. Laflamme, Phys. Lett. B 209, 39 (1988).

${ }^{14}$ G. V. Lavrelashvili, V. A. Rubakov, and P. G. Tinyakov, Nucl. Phys. B299, 757 (1988).

${ }^{15}$ S. W. Hawking, Phys. Lett. 134B, 403 (1984).

16J. J. Halliwell and R. Laflamme, Class. Quantum Grav. 6, 1839 (1989).

${ }^{17}$ R. C. Tolman, Relativity, Thermodynamics and Cosmology, (Dover, New York, 1987); S. W. Hawking, in Astrophysical Cosmology, edited by H. A. Bruck, G. V. Coyne, and M. S. Longair (Pontifica Academia Scientarium, Vatican City, 1982).
${ }^{18}$ P. J. Ruback, Report No. CERN-TH 5226/88, 1988 (unpublished).

${ }^{19}$ P. F. González-Díaz, Phys. Rev. D 40, 4184 (1989); J. Polchinski, University of Texas Report No. UTTG-06-89, 1989 (unpublished).

${ }^{20}$ A. Hosoya and T. Ogura, Phys. Lett. B 225, 117 (1989).

${ }^{21}$ B. J. Keay and R. Laflamme, Phys. Rev. D 40, 2118 (1989).

${ }^{22}$ P. F. González-Díaz, Phys. Lett. B 233, 117 (1989).

${ }^{23}$ R. C. Myers, Nucl. Phys. B323, 225 (1989).

${ }^{24}$ T. Padmanabhan, Ann. Phys. (N.Y.) 165, 38 (1985); P. F. González-Díaz, Ann. Phys. (Leipzig) 46, 309 (1989).

${ }^{25}$ T. Padmanabhan, Phys. Rev. D 28, 745 (1983); 28, 756 (1983).

${ }^{26}$ P. F. González-Diaz, Nucl. Phys. B (to be published).

${ }^{27}$ S. W. Hawking and D. N. Page, Phys. Rev. D 42, 2655 (1990).

${ }^{28}$ R. J. Glauber, Phys. Rev. 130, 2429 (1963).

${ }^{29}$ M. O. Scully and K. G. Whitney, in Progress in Optics, edited by E. Wolf (North-Holland, Amsterdam, 1972), Vol. 10.

${ }^{30}$ P. F. González-Díaz, Nuovo Cimento B 102, 195 (1988).

${ }^{31}$ I. Klebanov, L. Susskind, and T. Banks, Report No. SLACPUB-4705 1988 (unpublished); S. W. Hawking, Nucl. Phys. B335, 155 (1990).

${ }^{32}$ E. N. Lorenz, J. Atmos. Sci. 20, 130 (1963).

${ }^{33}$ B. Grinstein and M. Wise, Phys. Lett. B 212, 407 (1988).

${ }^{34}$ P. Candelas and S. Weinberg, Nucl. Phys. B237, 397 (1983).

35J. Anderson, in Eastern Theoretical Physics Conference, edited by M. E. Rose (Gordon and Breach, New York, 1963).

${ }^{36}$ A. D. Linde, Phys. Lett. 123B, 177 (1983).

${ }^{37}$ G. W. Gibbons, S. W. Hawking, and M. J. Perry, Nucl. Phys. B138, 141 (1978). 